\title{
Near infrared molecular hydrogen emission in the NGC 2264 IRS1 region
}

\author{
H. Wang ${ }^{1,2, \star}$, J. Yang ${ }^{1,2}$, M. Wang ${ }^{1,2}$, and J. Yan $^{1,2}$ \\ 1 Purple Mountain Observatory, Academia Sinica, Nanjing 210008, PR China \\ e-mail: jiyang@pmo.ac.cn; mwang@pmo.ac.cn; yanjun@pmo.ac.cn \\ 2 Chinese National Astronomical Observatories, Chinese Academy of Sciences, A20 Datun Road, \\ Chaoyang District, Beijing 100012, PR China
}

Received 22 January 2002 / Accepted 19 April 2002

\begin{abstract}
Molecular hydrogen $v=1-0 S(1)$ narrow band imaging revealed a cluster of 4, or probably 5, highly collimated $\mathrm{H}_{2}$ emission jets and many isolated $\mathrm{H}_{2}$ emission knots in the NGC 2264 IRS1 region. Jets 1 and 2 each consists of 1 bright and 2 faint knots. Jet 2 is shifted towards northeast relative to jet 1 , therefore, they are probably two distinct flows. Jet 3 is a short jet and jet 4 consists of 1 bright knot and several faint knots. Knots $I-K$ may constitute the fifth jet in the region. The relationship between these infrared outflows and the millimeter and submillimeter sources in the region, NGC 2264 MMS1-5, is discussed. Our detection of infrared outflows suggests that MMS1-5 are all in the protostar stage of their evolution. The high collimation of jets 1-4 may have important implication for the formation of intermediate and high mass stars.
\end{abstract}

Key words. ISM: jets and outflows - ISM: individual objects: NGC 2264 - stars: formation

\section{Introduction}

Outflows are an important tracer of ongoing star formation. They have manifestations in different wavelengths. For example, $\mathrm{CO}$ outflows observed in the millimeter wavelength trace the cold component of outflows (for a review see Bachiller 1996) while HH objects and near infrared molecular hydrogen emission, which have a typical spatial velocity of several hundred $\mathrm{km} \mathrm{s}^{-1}$, reveal the hot component (Reipurth \& Bally 2001). Due to the high extinction in the visual wavelength, however, optical observations can only reveal outflows slightly obscured. In contrast, molecular hydrogen emission at $2.122 \mu \mathrm{m}$ can reveal jets still embedded in cloud cores (see e.g. McCaughrean et al. 1994; Zinnecker et al. 1998), therefore, it can trace protostars which are at the very early stage of formation and are thus deeply embedded.

NGC 2264 consists of an open cluster of O- and B-type stars (Raimond 1966) at a distance of $760 \mathrm{pc}$ (Sung et al. 1997). Four groups of Herbig-Haro objects (Adams et al. 1979; Walsh et al. 1992) and numerous $\mathrm{H}_{\alpha}$ emission stars of T Tauri type (Herbig 1954) have been found

Send offprint requests to: H. Wang, e-mail: hcwang@pmo.ac.cn

* Visiting astronomer at the Astrophysical Institute and University Observatory, Friedrich-Schiller-University, Schillergaesschen 2-3, 07745 Jena, Germany. in the region. Observations of molecular lines (Crutcher et al. 1978) have revealed a dense molecular cloud of $\sim 2 \times 10^{4} M_{\odot}$, extending roughly in the north-south direction. An ammonia survey of NGC 2264 revealed two main components of the cloud, each having complex structure (Krügel et al. 1987). Both of the subclouds coincide with peaks of far-infrared emission (Sargent et al. 1984; Schwartz et al. 1985). The stronger, southern peak coincides with the Allen's infrared source IRS1, which is believed to be a $9.5 M_{\odot}$ B2 zero-age main-sequence star (Allen 1972).

Evidence of ongoing star formation in the NGC 2264 IRS1 region has been provided by different observations. Red-shifted, high-velocity gas was detected in CO (Margulis et al. 1988), with a peak in the red-shifted emission some 30 arcsec to the south of IRS1. Recently, Schreyer et al. (1997) carried out a detailed molecular line study of this region and identified two bipolar outflows, one associated with IRS1, and one associated with a deeply embedded cluster they observed in this region in the near-infrared $K$-band. Using the HST NICMOS camera Thompson et al. (1998) identified six new sources in the vicinity of IRS1 as pre-main-sequence stars. More recently, Ward-Thompson et al. (2000) conducted submillimeter and millimeter continuum observations of the region of IRS1 and detected a cluster 


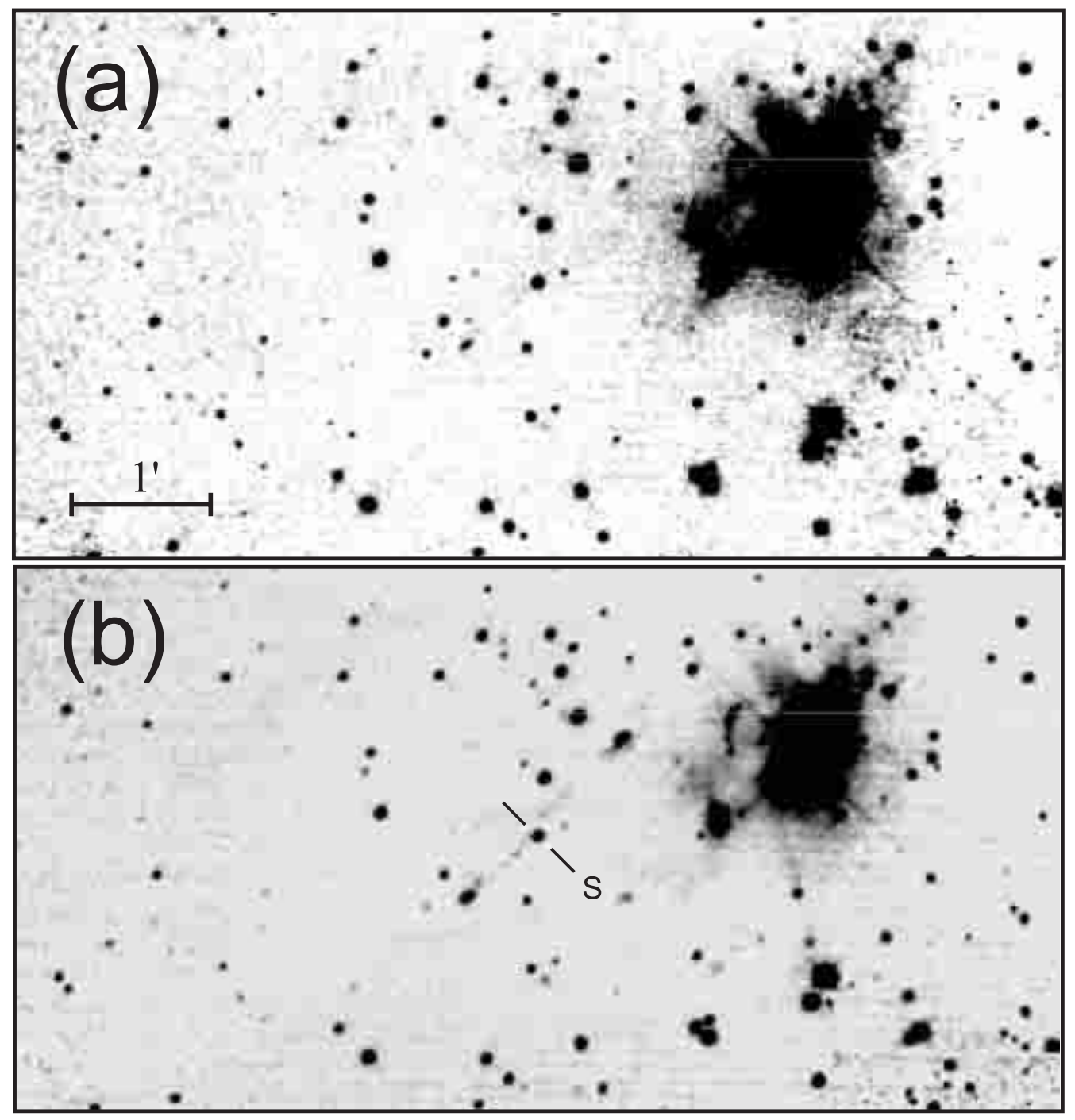

Fig. 1. Near-infrared images of the NGC 2264 IRS1 region, a) in $K^{\prime}$ band, b) in $\mathrm{H}_{2}$ band. The $\mathrm{H}_{\alpha}$ emission star "S" is indicated in b). The fields are $7^{\prime} .7 \times 4.0$; north is up, east is to the left.

of 5 submillimeter sources, NGC 2264 MMS1-5. Though MMS3 was identified as the driving source of one the CS outflows in the region, the evolutionary status of the other submillimeter sources is unclear. In this paper we present our near infrared studies of the region in order to search for new outflows and to determine the revolutionary stages of the submillimeter sources.

\section{Observations}

The near infrared imaging observations were carried out on 1999 November 20 and 25 at the $1.88 \mathrm{~m}$ telescope in Okayama Astronomical Observatory, Japan. The infrared camera OASIS (Yamashita et al. 1995), which is equipped with a near-infrared camera and multi-object spectrometer (NICMOS3) array, was used for the observations. The field of view of OASIS is $4.2 \times 4.2$ with a plate scale of $0.97 /$ pixel. Dithered $J, H, K^{\prime}(2.16 \mu \mathrm{m})$ and $\mathrm{H}_{2}$ $v=1-0 S(1)(2.12 \mu \mathrm{m})$ images were obtained and the total exposures are 220, 40, 260, and 2280 s respectively.
The weather conditions during the observations were good and the seeing was around 2 .' 0 . The images were darksubtracted, flat field-normalized, sky frame-subtracted, registered, and combined using the IRAF package. The flat field was constructed with two sets of dome flat frames taken by switching an illuminating lamp on and off. The sky frame was obtained by median filtering the target images. The standard stars used for photometry is AS 11 (Hunt et al. 1998).

\section{Results}

In Fig. 1 we present the images of the region in $K^{\prime}$ and $\mathrm{H}_{2}$ bands (panels a and b). The continuum subtracted $\mathrm{H}_{2}$ image is presented in Fig. 2. In Fig. 2 superposed to the image are the outlines of the CS outflows detected by Schreyer et al. (1997). The positions of the submillimeter sources detected by Ward-Thompson et al. (2000) are marked with large pluses. The position of the brightest star in the small cluster detected by Schreyer et al. (1997) 


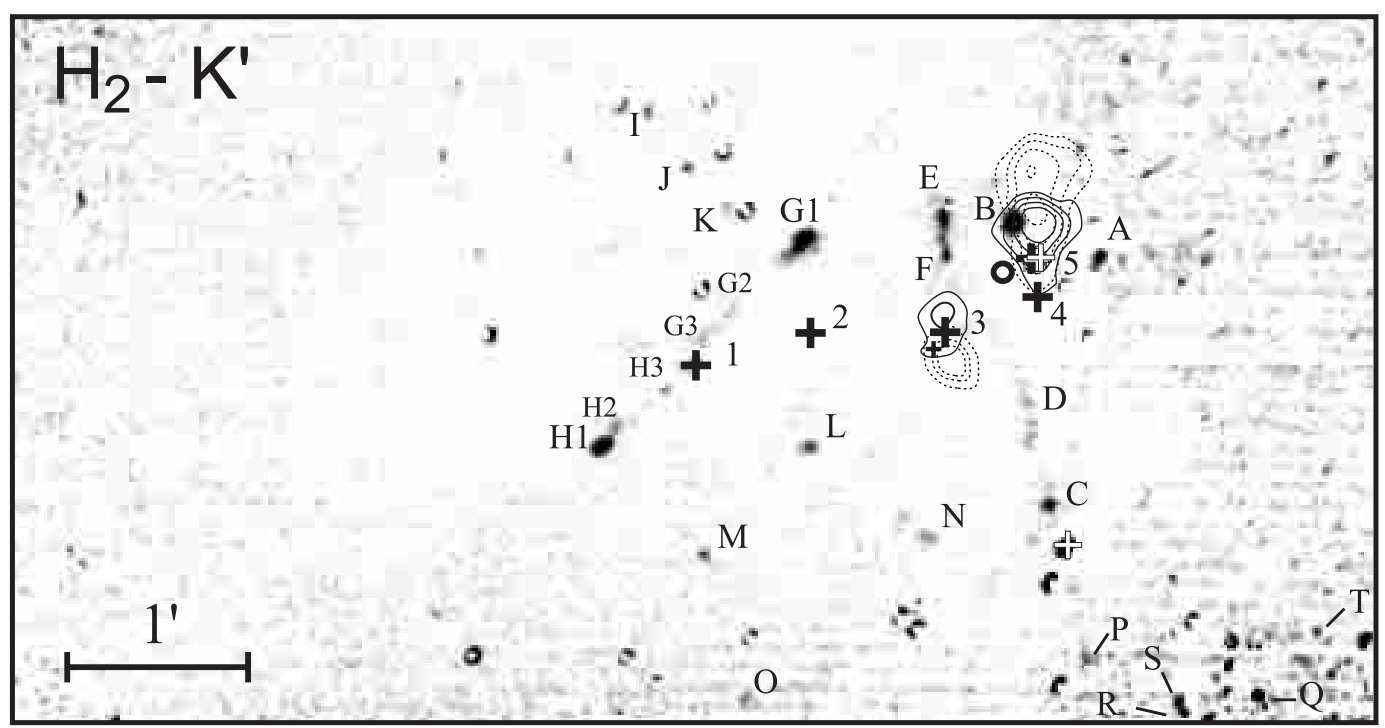

Fig. 2. The net $\mathrm{H}_{2}$ emission image. The outlines are the CS outflows from Schreyer et al. (1997). The positions of millimeter and submillimeter sources MMS1-5 from Ward-Thompson et al. (2000) are marked with large pluses and the position of the small embedded cluster detected by Schreyer et al. (1997) is marked with a small plus. The open circle near MMS4-5 indicates the position of IRAS $06384+0932$. The white pluses mark the stars saturated in $\mathrm{K}^{\prime}$ image, including IRS1. All the others are the same as in Fig. 1.

is marked with a small plus. From Fig. 2 it can be seen that there are abundant $\mathrm{H}_{2}$ emission in the region. All the emission is relatively compact. Therefore, they should be shock excited. The emission knots are labeled in Fig. 2 with A-T. Two stars including IRS1 are saturated in the $K^{\prime}$ image, and thus are not completely subtracted from the $\mathrm{H}_{2}$ image. In Fig. 2 the residual of these two stars are marked with white pluses. The coordinates of the emission knots, which are determined using the GSC catalog, are listed in Table 1 . The accuracy of these determinations is within $1^{\prime \prime}$ both in RA and Dec.

From Fig. 2 one can see that knots H1-3 align well with each other and constitute a jet of high collimation, here designated jet 1 , in the direction of $\mathrm{PA} \simeq 130^{\circ}$. Jet 1 aligns well with star "S" (see Fig. 1b). The optical low dispersion spectrum of star "S", taken with the Beijing Astronomical Observatory $2.16 \mathrm{~m}$ telescope on 1999 Dec. 8, is presented in Fig. 3. From Fig. 3 we can see that star " $\mathrm{S}$ " has strong $\mathrm{H}_{\alpha}$ emission with an equivalent width of $38.2 \AA$, indicating that it's a young star. Knots G1-3 also align well with each other and constitute another jet of high collimation (jet 2) in the direction of $\mathrm{PA} \simeq 134^{\circ}$. Although jet 2 looks like the counterpart of jet 1 , one can see that, with respect to the axis of jet 1 the axis of jet 2 is apparently shifted towards the northeast. Therefore they are probably two distinct flows. Knots E and $\mathrm{F}$ constitute a short jet (jet 3) in the orientation of nearly north-south. About 1.5 southwest of jet 3 are the bright knot $\mathrm{C}$ and the faint patch $\mathrm{D}$. Patch $\mathrm{D}$ consists of several faint knots. It can be seen that knot $\mathrm{C}$ and patch $\mathrm{D}$ constitute another jet (jet 4) in the region. To the west of jet 3 is knot $\mathrm{B}$, the brightest knot of the region. Knot $\mathrm{B}$ is located along the axis of jet 4 and may be part of jet 4 . To the northeast of jet 2 there are 3 isolated knots, $I-K$, which align well with each other and may constitute a jet (see the discussion below). The isolated knots L-O are located in the southern part of the region. In the southwestern corner of Fig. 2, $\mathrm{H}_{2}$ emission knots P-T can be identified although the $S / N$ ratio there is relatively low.

\section{Discussions}

At 350, 450, 800 and $1300 \mu \mathrm{m}$, Ward-Thompson et al. (2000) detected a cluster of 5 submillimeter sources, NGC 2264 MMS1-5, in the IRS1 region. The masses of the sources lie in the range $\sim 10-50 M_{\odot}$. They suggest that each of the sources is forming one or more intermediate or high mass stars. From Fig. 2 we can see that submillimeter source MMS1 coincides very well with the young star "S". However, the near infrared colors of star "S" from our photometry are $J-H=1.09$ and $H-K^{\prime}=0.31$, indicating that star " $\mathrm{S}$ " is not deeply embedded in dense gas $\left(A_{\mathrm{v}} \simeq 2.5\right)$. Millimeter and submillimeter observations indicate that the column density towards MMS1 is $2.5 \times 10^{23} \mathrm{~cm}^{-2}$ which corresponds to an extinction of $A_{\mathrm{v}} \simeq 125$. Therefore, the coincidence of MMS1 with star "S" should be by chance. The close association and good alignment of MMS1 and star "S" with jet 1 suggest that one of these sources drives the jet. Although jets 1 and 2 have nearly the same position angle, one can see from Fig. 2 that the axis of jet 2 is shifted to the northeast with respect to that of jet 1 . As both jets 1 and 2 have a high collimation, the axis shift can not be explained by a deflection due to interaction between the jets and the gas clumps in the surrounding medium. Therefore, they should represent two distinct outflows. Obviously, star " $\mathrm{S}$ " is not the driving source of jet 2 . Taking into account the relatively large millimeter and submillimeter observation beams, it 


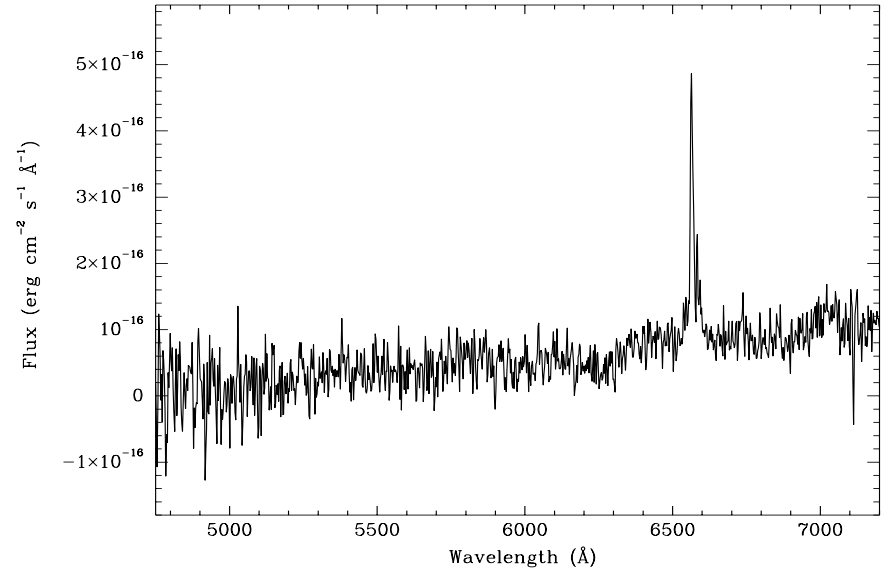

Fig. 3. Low dispersion spectrum of the star "S".

is possible that MMS1 drives jet 2. The close association of MMS1 with 2 highly collimated jets suggests that MMS1 is at the star forming stage of its evolution rather than to be a pre-stellar core as postulated by Ward-Thompson et al. (2000).

Jet 3 (knots E and F) is a short but highly collimated jet. It points to the submillimeter source, NGC 2264 MMS3. From Fig. 2, as noted by Ward-Thompson et al. (2000), the CS outflow (Schreyer et al. 1997) is located more symmetrically with respect to MMS3 than with the embedded cluster. The morphology of jet 3 suggests that it is also originated from MMS3. Therefore, our infrared data support the suggestion that MMS3 is at the star forming stage.

Jet 4 (Knot $\mathrm{C}$ and patch $\mathrm{D})$ is oriented in the direction of $\mathrm{PA} \simeq 12^{\circ}$ and points towards IRAS 06384+0932 (see Fig. 2). Submillimeter sources MMS4-5 and the near infrared source IRS 1 are all located within the error ellipse of IRAS $06384+0932$. All these objects are the possible driving source of jet 4 . In addition, jet 4 may be associated with the CS outflow of IRS1.

We note that knots $I-K$ align well with each other. We postulate that they constitute another jet (jet 5) in the region. Knots $I-K$ align well with MMS2, so it is possible that jet 5 is driven by MMS2.

In summary, a cluster of 4 , or probably 5 , highly collimated near infrared $\mathrm{H}_{2}$ emission jets are detected in the NGC 2264 IRS1 region. Millimeter and submillimeter observations suggest that MMS1-5 each are forming one or multiple intermediate or high mass stars (WardThompson et al. 2000). The near infrared outflows detected in this study suggest that MMS1-5 have reached the protostar stages of their evolution. Outflows from massive protostars are usually observed to be of lower collimation than their counterparts driven by low mass stars (e.g. Richer et al. 2000). Our detection of a cluster of highly collimated outflows in the NGC 2264 IRS1 region may have important implication for the formation of intermediate and high mass stars.

Acknowledgements. We thank the staff members of Okayama Astronomical Observatory, Japan and the staff members of
Table 1. A List of $\mathrm{H}_{2}$ emission in the NGC 2264 IRS1 region.

\begin{tabular}{lccl}
\hline \hline Object & $\alpha(1950)$ & $\delta(1950)$ & Comments \\
\hline A & 63823.90 & 93228.7 & Bright knot \\
B & 63825.84 & 93240.5 & Bright knot \\
C & 63825.18 & 93106.7 & Bright knot \\
D & 63825.64 & 93141.0 & Faint patch \\
E & 63827.44 & 93242.9 & Bright elongated knot \\
F & 63827.38 & 93231.2 & Bright elongated knot \\
G1 & 63830.56 & 93237.6 & Bright elongated knot \\
G2 & 63832.34 & 93212.5 & Faint knot \\
G3 & 63832.86 & 93206.1 & Faint knot \\
H1 & 63835.22 & 93130.4 & Bright elongated knot \\
H2 & 63834.87 & 93136.5 & Knot \\
H3 & 63833.73 & 93148.6 & Knot \\
I & 63833.97 & 93320.8 & Knot \\
J & 63833.11 & 93302.1 & Knot \\
K & 63832.22 & 93246.3 & Knot \\
L & 63830.55 & 93128.1 & Knot \\
M & 63832.95 & 93053.0 & Knot \\
N & 63827.96 & 93057.3 & Knot \\
O & 63832.14 & 93003.9 & Knot \\
P & 63824.45 & 93015.4 & Knot \\
Q & 63820.59 & 93001.7 & Knot \\
R & 63822.26 & 92956.5 & Knot \\
S & 63822.40 & 93000.3 & Knot \\
T & 63819.23 & 93022.2 & Knot \\
\hline
\end{tabular}

Beijing Astronomical Observatory for their excellent support during observations of this work. We thank Yongqiang Yao and Zhibo Jiang for their help during the near infrared observations. We are grateful to the referee, P. Hartigan, for his comments which improve the manuscript. This research was supported by NSFC grants 10073021, 19625307 and G19990754.

\section{References}

Adams, M. T., Strom, K. M., \& Strom, S. E. 1979, ApJ, 230, L183

Allen, D. A. 1972, ApJ, 172, L55

Bachiller, R. 1996, ARA\&A, 34, 111

Crutcher, R. M., Hartkopf, W. I., \& Giguere, P. T. 1978, ApJ, 226,839

Herbig, G. H. 1954, ApJ, 119, 483

Hunt, L. K., Mannucci, F., Testi, L., et al. 1998, AJ, 115, 2594

Krügel, E., Güsten, R., Schulz, A., \& Thum, C. 1987, A\&A, 185,283

Margulis, M., Lada, C. J., \& Snell, R. L. 1988, ApJ, 333, 316

McCaughrean, M. J., Rayner, J. T., \& Zinnecker, H. 1994, ApJ, 436, L189

Raimond, E. 1966, Bull. Astron. Inst. Netherlands, 18, 191

Reipurth, B., \& Bally, J. 2001, ARA\&A, 39, 403 
Richer, J., Shepherd, D., Cabrit, S., Bachiller, R., \& Churchwell, E. 2000, in Protostars and Planets IV, ed. V. Mannings, A. P. Boss, \& S. S Russell (Tucson: Univ. Arizona Press)

Sargent, A. I., van Duinen, R. J., Nordh, H. L., et al. 1984, A\&A, 135, 377

Schreyer, K., Helmich, F. P., van Dishoek, E. F., \& Henning, Th. 1997, A\&A, 326, 347

Schwartz, P. R., Thronson, H. A. Jr., Odenwald, S. F., et al. 1985, ApJ, 292, 231

Thompson, R. I., Corbin, M. R., Young, E., \& Schneider, G. 1998, ApJ, 492, L177
Sung, H., Bessell, M. S., \& Lee, S. 1997, AJ, 114, 2644

Walsh, J. R., Ogura, K., \& Reipurth, Bo 1992, MNRAS, 257, 110

Ward-Thompson, D., Zylka, R., Mezger, P. G., \& Sievers, A. W. 2000, A\&A, 355, 1122

Yamashita, T., Nishihara, E., Okumura, S., Mori, A., \& Watanabe, E. 1995, in Scientific and Engineering Frontiers for 8-10 m Telescopes, ed. M. Iye, \& T. Nishimura (Tokyo: Univ. Acad.), 285

Zinnecker, H., McCaughrean, M. J., \& Rayner, J. T. 1998, Nature, 394, 862 